\title{
The Virasoro Algebra and Some Exceptional Lie and Finite Groups ${ }^{\star}$
}

\author{
Michael P. TUITE
}

Department of Mathematical Physics, National University of Ireland, Galway, Ireland

E-mail: Michael.Tuite@nuigalway.ie

Received October 09, 2006, in final form December 16, 2006; Published online January 08, 2007

Original article is available at http://www.emis.de/journals/SIGMA/2007/008/

\begin{abstract}
We describe a number of relationships between properties of the vacuum Verma module of a Virasoro algebra and the automorphism group of certain vertex operator algebras. These groups include the Deligne exceptional series of simple Lie groups and some exceptional finite simple groups including the Monster and Baby Monster.
\end{abstract}

Key words: vertex operator algebras; Virasoro algebras; Deligne exceptional series; Monster group

2000 Mathematics Subject Classification: 17B68; 20D08; 17B69; 81R05; 81R10

\section{Introduction}

This paper is based on a talk given at the Lochlainn O'Raifeartaigh Symposium on NonPerturbative and Symmetry Methods in Field Theory, Budapest, 2006. We describe a number of relationships between the vacuum Verma module of a Virasoro algebra and the Deligne exceptional series of Lie algebras and also some exceptional finite groups such as the Monster and Baby Monster. The setting in which this is explained is the theory of Vertex Operator Algebras (VOAs) or chiral conformal field theory. In particular, we construct certain Casimir vectors that are invariant under the VOA automorphism group. If these vectors are elements of the vacuum Verma module then the VOA Lie or Griess algebra structure is constrained. This is an idea originally introduced by Matsuo for Griess algebras [11] and further developed for Lie algebras in [12]. Our approach is more general with weaker assumptions and is based on a consideration of a certain rational correlation function. The constraints on the Lie or Griess algebra structure arise from an analysis of the expansion of this correlation function in various domains. The paper contains a quite elementary description of the main ingredients where many of the detailed proofs are omitted. These will appear elsewhere [15].

\section{The Virasoro algebra, Verma modules and the Kac determinant}

We begin with a number of basic definitions and properties for Virasoro algebras e.g. [9]. The Virasoro Algebra Vir with central charge $C$ is given by

$$
\left[L_{m}, L_{n}\right]=(m-n) L_{m+n}+\left(m^{3}-m\right) \frac{C}{12} \delta_{m,-n}, \quad\left[L_{m}, C\right]=0 .
$$

${ }^{\star}$ This paper is a contribution to the Proceedings of the O'Raifeartaigh Symposium on Non-Perturbative and Symmetry Methods in Field Theory (June 22-24, 2006, Budapest, Hungary). The full collection is available at http://www.emis.de/journals/SIGMA/LOR2006.html 
The Vacuum Verma Module $V(C, 0)$ for Vir is defined as follows. Let $\mathbf{1} \in V(C, 0)$ denote the vacuum vector where

$$
L_{n} \mathbf{1}=0, \quad n \geq-1 .
$$

Then $V(C, 0)$ is the set of Virasoro descendents of the vacuum (highest weight) vector

$$
V(C, 0)=\mathbb{C}\left[L_{-n_{1}} L_{-n_{2}} \cdots L_{-n_{k}} \mathbf{1} \mid n_{1} \geq n_{2} \geq \cdots \geq n_{k} \geq 2\right] .
$$

$V(C, 0)$ is a module for Vir graded by $L_{0}$ where

$$
L_{0}\left(L_{-n_{1}} \cdots L_{-n_{k}} \mathbf{1}\right)=n L_{-n_{1}} \cdots L_{-n_{k}} \mathbf{1},
$$

and where $n=n_{1}+\cdots+n_{k} \geq 0$ is called the (Virasoro) level. Then

$$
V(C, 0)=\bigoplus_{n \geq 0} V^{(n)}(C, 0),
$$

where $V^{(n)}(C, 0)$ denotes the vectors of level $n$. Clearly $\operatorname{dim} V^{(n)}(C, 0)<\infty$ for all $n$.

In general we may consider a Verma Module $V(C, h)$ defined in terms of highest weight vector $v \in V(C, h)$ obeying

$$
L_{n} v=h \delta_{n, 0} v, \quad n \geq 0 .
$$

$v$ is called a primary vector of level $h$. Then $V(C, h)$ is generated by the Virasoro descendents of $v$. However, we are primarily interested only in the vacuum module $V(C, 0)$ here.

$V(C, 0)$ is an irreducible module provided no descendent vector is itself a primary vector. The irreducibility of $V(C, 0)$ can be established by considering the Kac determinant defined as follows. Define a symmetric bilinear form $\langle\cdot, \cdot\rangle$ on $V(C, 0)$ with

$$
\left\langle L_{-n} u, v\right\rangle=\left\langle u, L_{n} v\right\rangle,
$$

for $u, v \in V(C, 0)$ and normalization $\langle\mathbf{1}, \mathbf{1}\rangle=1$. Note that $\langle u, v\rangle=0$ for $u, v$ of different Virasoro level. Thus we consider the level $n$ Gram matrix

$$
M^{(n)}=(\langle u, v\rangle),
$$

for vacuum descendents $u, v \in V^{(n)}(C, 0)$. Then $V(C, 0)$ is irreducible iff the Kac determinant $\operatorname{det} M^{(n)} \neq 0$ (with a similar formulation for $V(C, h)$ ) [9].

There exists a well-known general product formula for $\operatorname{det} M^{(n)}$ due to Kac and proved by Feigin and Fuchs [9]. However, for our purposes, it is useful to explicitly $\operatorname{display} \operatorname{det} M^{(n)}$ for even levels $n \leq 10$. At level $n=2$ we have $V^{(2)}(C, 0)=\mathbb{C} \omega$ where $\omega=L_{-2} \mathbf{1}$ is called the Conformal Vector. Then

$$
\operatorname{det} M^{(2)}=\langle\omega, \omega\rangle=\left\langle\mathbf{1}, L_{2} L_{-2} \mathbf{1}\right\rangle=\frac{C}{2} .
$$

Thus $C \neq 0$ for irreducible $V(C, 0)$.

At level 4 we have $V^{(4)}(C, 0)=\mathbb{C}\left[L_{-2} L_{-2} \mathbf{1}, L_{-4} \mathbf{1}\right]$ with

$$
M^{(4)}=\left[\begin{array}{cc}
C\left(4+\frac{1}{2} C\right) & 3 C \\
3 C & 5 C
\end{array}\right], \quad \operatorname{det} M^{(4)}=\frac{1}{2} C^{2}(5 C+22) .
$$

Thus $C \neq 0,-22 / 5$ for irreducible $V(C, 0)$.

Similarly we may compute $\operatorname{det} M^{(n)}$ for $n=6,8,10$ to find

$$
\begin{aligned}
& \operatorname{det} M^{(6)}=\frac{3}{4} C^{4}(5 C+22)^{2}(2 C-1)(7 C+68), \\
& \operatorname{det} M^{(8)}=3 C^{7}(5 C+22)^{4}(2 C-1)^{2}(7 C+68)^{2}(3 C+46)(5 C+3), \\
& \operatorname{det} M^{(10)}=\frac{225}{2} C^{12}(5 C+22)^{8}(2 C-1)^{5}(7 C+68)^{4}(3 C+46)^{2}(5 C+3)^{2}(11 C+232) .
\end{aligned}
$$

where $\operatorname{dim} V^{(6)}(C, 0)=4, \operatorname{dim} V^{(8)}(C, 0)=7$ and $\operatorname{dim} V^{(10)}(C, 0)=12$. 


\section{Some exceptional group numerology}

Let us consider the prime factors of the Kac determinant $\operatorname{det} M^{(n)}$ for level $n \leq 10$ for particular values of $C$. We observe some coincidences with properties of a number of exceptional Lie and finite groups. Later on we will explain the underlying reason for these coincidences and obtain many other relationships with the Virasoro structure.

Deligne's exceptional Lie algebras $A_{1}, A_{2}, G_{2}, D_{4}, F_{4}, E_{6}, E_{7}, E_{8}$. This set of simple Lie algebras has been shown recently to share a surprising number of representation theory properties in common [2]. For example, the dimension of the adjoint representation $d$ of each of these algebras can be expressed in terms of the dual Coxeter number $h^{\vee}$ for the given algebra (as originally found by Vogel):

$$
d=\frac{2\left(5 h^{\vee}-6\right)\left(h^{\vee}+1\right)}{h^{\vee}+6} .
$$

Let us compare $d$ to the independent factors $C$ and $5 C+22$ of the level 4 Kac determinant $\operatorname{det} M^{(4)}$ for particular values of $C$ :

Table 1.

\begin{tabular}{|c|c|c|c|c|c|c|c|c|}
\hline & $A_{1}$ & $A_{2}$ & $G_{2}$ & $D_{4}$ & $F_{4}$ & $E_{6}$ & $E_{7}$ & $E_{8}$ \\
\hline$h^{\vee}$ & 2 & 3 & 4 & 6 & 9 & 12 & 18 & 30 \\
\hline$d$ & 3 & $2^{3}$ & $2 \cdot 7$ & $2^{2} \cdot 7$ & $2^{2} \cdot 13$ & $2 \cdot 3 \cdot 13$ & $7 \cdot 19$ & $2^{3} \cdot 31$ \\
\hline$C$ & 1 & 2 & $\frac{2 \cdot 7}{5}$ & $2^{2}$ & $\frac{2 \cdot 13}{5}$ & $2 \cdot 3$ & 7 & $2^{3}$ \\
\hline $5 C+22$ & $3^{3}$ & $2^{5}$ & $2^{2} \cdot 3^{2}$ & $2 \cdot 3 \cdot 7$ & $2^{4} \cdot 3$ & $2^{2} \cdot 13$ & $3 \cdot 19$ & $2 \cdot 31$ \\
\hline
\end{tabular}

The first row of Table 1 shows $h^{\vee}$ whereas the second row shows the prime factorization of $d$ for each algebra. The third row shows the prime factorization for particular values of $C$ (where $C$ is the rank of the algebra for the simply-laced cases $A_{1}, A_{2}, D_{4}, E_{6}, E_{7}, E_{8}$ ). Notice that each prime divisor of $d$ is a prime divisor of the numerator of either $C$ or $5 C+22$ and hence the numerator of $\operatorname{det} M^{(4)}$.

Some prime divisors of the order of a number of exceptional finite groups are also related to the Kac determinant factors. We highlight three examples.

The Monster simple group $\mathbb{M}$. The classification theorem of finite simple groups states that a finite simple group is either one of several infinite families of simple groups (e.g. the alternating groups $A_{n}$ for $n \geq 5$ ) or else is one of 26 sporadic finite simple groups. The largest sporadic group is the Monster group $\mathbb{M}$ of (prime factored) order

$$
|\mathbb{M}|=2^{46} \cdot 3^{20} \cdot 5^{9} \cdot 7^{6} \cdot 11^{2} \cdot 13^{3} \cdot 17 \cdot 19 \cdot 23 \cdot 29 \cdot 31 \cdot 41 \cdot 47 \cdot 59 \cdot 71 .
$$

The two lowest dimensional non-trivial irreducible representations are of dimension $d_{2}=196883$ $=47 \cdot 59 \cdot 71$ and $d_{3}=21296876=2^{2} \cdot 31 \cdot 41 \cdot 59 \cdot 71$. Consider the independent factors of $\operatorname{det} M^{(10)}$ for $C=24$

Table 2.

\begin{tabular}{|c|c|c|c|c|c|c|}
\hline$C$ & $5 C+22$ & $2 C-1$ & $7 C+68$ & $3 C+46$ & $5 C+3$ & $11 C+232$ \\
\hline $2^{3} \cdot 3$ & $2 \cdot 71$ & 47 & $2^{2} \cdot 59$ & $2 \cdot 59$ & $3 \cdot 41$ & $2^{4} \cdot 31$ \\
\hline
\end{tabular}

Notice that the prime divisors $2,31,41,47,59,71$ of $|\mathbb{M}|$ (which are all of the prime divisors of $d_{2}$ and $\left.d_{3}\right)$ are divisors of $\operatorname{det} M^{(10)}$.

The Baby Monster simple group $\mathbb{B}$. The second largest sporadic group is the Baby Monster group $\mathbb{B}$ of order

$$
|\mathbb{B}|=2^{41} \cdot 3^{13} \cdot 5^{6} \cdot 7^{2} \cdot 11 \cdot 13 \cdot 17 \cdot 19 \cdot 23 \cdot 31 \cdot 47 .
$$


The prime divisors $2,3,5,23,31,47$ of $|\mathbb{B}|$ are divisors of the numerator of the independent factors of $\operatorname{det} M^{(6)}$ for $C=23 \frac{1}{2}$ :

Table 3.

\begin{tabular}{|c|c|c|c|}
\hline$C$ & $5 C+22$ & $2 C-1$ & $7 C+68$ \\
\hline$\frac{47}{2}$ & $\frac{3^{2} \cdot 31}{2}$ & $2 \cdot 23$ & $\frac{3 \cdot 5 \cdot 31}{2}$ \\
\hline
\end{tabular}

The simple group $O_{10}^{+}(2)$. This group has order

$$
\left|O_{10}^{+}(2)\right|=2^{20} \cdot 3^{5} \cdot 5^{2} \cdot 7 \cdot 17 \cdot 31 .
$$

The prime divisors $2,3,5,31$ of $\left|O_{10}^{+}(2)\right|$ are divisors of the independent factors of $\operatorname{det} M^{(6)}$ for $C=8$

Table 4.

\begin{tabular}{|c|c|c|c|}
\hline$C$ & $5 C+22$ & $2 C-1$ & $7 C+68$ \\
\hline $2^{3}$ & $2 \cdot 31$ & $3 \cdot 5$ & $2^{2} \cdot 31$ \\
\hline
\end{tabular}

\section{Vertex operator algebras}

\subsection{Axioms}

The observations made in the last section can be understood in the context of Vertex Operator Algebras (VOAs) $[1,8,13]$. The basic idea is that the groups appearing above arise as automorphism (sub)groups of particular VOAs with the given central charge $C$. In this section we review the relevant aspects of VOA theory required in the subsequent sections.

A Vertex Operator Algebra (VOA) consists of a $\mathbb{Z}$-graded vector space $V=\bigoplus_{k \geq 0} V^{(k)}$ with $\operatorname{dim} V^{(k)}<\infty$ and with the following properties:

- Vacuum. $V^{(0)}=\mathbb{C} \mathbf{1}$ for vacuum vector $\mathbf{1}$.

- Vertex operators (state-field correspondence). For each $a \in V^{(k)}$ we have a vertex operator

$$
Y(a, z)=\sum_{n \in \mathbb{Z}} a_{n} z^{-n-k}
$$

with component operators (modes) $a_{n} \in \operatorname{End} V$ such that

$$
\left.Y(a, z) \cdot \mathbf{1}\right|_{z=0}=a_{-k} \cdot \mathbf{1}=a .
$$

$z$ is a formal variable here (but is taken as a complex number in physics). Note also that we are employing "physics modes" in (4.1).

The vacuum vector has vertex operator

$$
Y(\mathbf{1}, z)=I d_{V}
$$

so that $\mathbf{1}_{n}=\delta_{n, 0}$.

- Virasoro subalgebra. There exists a conformal vector $\omega \in V^{(2)}$ with

$$
Y(\omega, z)=\sum_{n \in \mathbb{Z}} L_{n} z^{-n-2}
$$


where the modes $L_{n}$ form a Virasoro algebra (2.1) of central charge $C$. The $\mathbb{Z}$-grading is determined by $L_{0}$ with $V^{(k)}=\mathbb{C}\left[a \in V \mid L_{0} a=k a\right]$.

$L_{-1}$ acts as a translation operator with

$$
Y\left(L_{-1} a, z\right)=\partial_{z} Y(a, z) \text {. }
$$

- Locality. For any pair of vertex operators we have for sufficiently large integer $N$

$$
(x-y)^{N}[Y(a, x), Y(b, y)]=0 .
$$

These axioms lead to the following basic VOA properties: e.g. [8, 13].

Translation. For any $a \in V$ then for $|y|<|x|$ (i.e. formally expanding in $y / x$ )

$$
e^{y L_{-1}} Y(a, x) e^{-y L_{-1}}=Y(a, x+y) .
$$

Skew-symmetry. For $a, b \in V$ then

$$
Y(a, z) b=e^{z L_{-1}} Y(b,-z) a .
$$

Associativity. For $a, b \in V$ then for $|x-y|<|y|<|x|$ we find $^{1}$

$$
Y(a, x) Y(b, y)=Y(Y(a, x-y) b, y) .
$$

Borcherds' commutator formula. For $a \in V^{(k)}$ and $b \in V$ then

$$
\left[a_{m}, b_{n}\right]=\sum_{j \geq 0}\left(\begin{array}{c}
m+k-1 \\
j
\end{array}\right)\left(a_{j-k+1} b\right)_{m+n} .
$$

Example. For $a=\omega \in V^{(2)}$ and $m=0$ we have $\left[L_{0}, b_{n}\right]=\left(L_{-1} b\right)_{n}+\left(L_{0} b\right)_{n}=-n b_{n}$. Thus

$$
b_{n}: V^{(m)} \rightarrow V^{(m-n)} .
$$

In particular, the zero mode $b_{0}$ is a linear operator on $V^{(m)}$. Similarly, for all $m$ and any primary vector $b \in V^{(h)}$, we have the familiar property from conformal field theory

$$
\left[L_{m}, b_{n}\right]=((h-1) m-n) b_{m+n} .
$$

\subsection{The Li-Zamolodchikov metric}

Assume that $V^{(0)}=\mathbb{C} \mathbf{1}$ and $L_{1} v=0$ for all $v \in V^{(1)}$. Then there exists a unique invariant bilinear form $\langle\cdot, \cdot\rangle$ where for all $a, b, c \in V[4,10]$

$$
\left\langle Y\left(e^{z L_{1}}\left(-\frac{1}{z^{2}}\right)^{L_{0}} c, \frac{1}{z}\right) a, b\right\rangle=\langle a, Y(c, z) b\rangle,
$$

and with normalization $\langle\mathbf{1}, \mathbf{1}\rangle=1$. For any quasi-primary $c \in V^{(k)}$ (i.e. where $L_{1} c=0$ ) then

$$
\left\langle c_{n} a, b\right\rangle=(-1)^{k}\left\langle a, c_{-n} b\right\rangle .
$$

Choosing $a \in V^{(k)}, n=k$ and $b=\mathbf{1}$, (4.9) implies for quasi-primary $c$

$$
c_{k} a=(-1)^{k}\langle a, c\rangle \mathbf{1} \text {. }
$$

For $c=\omega,(4.9)$ also implies

$$
\left\langle L_{n} a, b\right\rangle=\left\langle a, L_{-n} b\right\rangle .
$$

$\langle\cdot, \cdot\rangle$ is symmetric [4] and, furthermore, is non-degenerate iff $V$ is semisimple [10]. We call such a unique non-degenerate form the Li-Zamolodchikov or Li-Z metric. In particular, considering the Li-Z metric on the vacuum Virasoro descendents $V(C, 0) \subset V$ implies that the Kac determinant $\operatorname{det} M^{(n)} \neq 0$ for each level $n$.

\footnotetext{
${ }^{1}$ Strictly speaking, (4.5) holds for matrix elements taken with repect to any pair $u^{\prime}, v$ for any $v \in V$ and $u^{\prime} \in V^{\prime}$, the dual vector space [4].
} 


\subsection{Lie and Griess algebras}

Lie algebras. Let us consider a number of relevant subalgebras of a VOA $V$ with Li-Z metric. Suppose that $\operatorname{dim} V^{(1)}>0$ and define for all $a, b \in V^{(1)}$

$$
\operatorname{ad}(a) b=a_{0} b=-b_{0} a,
$$

where the second equality follows from skew-symmetry (4.4). We may thus define a bracket $[a, b]=\operatorname{ad}(a) b$ which satisfies the Jacobi identity so that $V^{(1)}$ forms a Lie algebra. Furthermore, the Li-Z metric $\langle a, b\rangle$ on $V^{(1)}$ is an invariant non-degenerate symmetric bilinear form with ${ }^{2}$

$$
\langle[a, b], c\rangle=\left\langle-b_{0} a, c\right\rangle=\left\langle a, b_{0} c\right\rangle=\langle a,[b, c]\rangle,
$$

for all $a, b, c \in V^{(1)}$. The Borcherds' commutator formula (4.6) then defines a Kac-Moody algebra $^{3}$

$$
\left[a_{m}, b_{n}\right]=\left(a_{0} b\right)_{m+n}+\left(a_{1} b\right)_{m+n}=[a, b]_{m+n}-m\langle a, b\rangle \delta_{m+n, 0},
$$

using $a_{1} b=-\langle a, b\rangle \mathbf{1}$ from (4.10).

Griess algebras. Suppose that $\operatorname{dim} V^{(1)}=0$ and consider $a, b \in V^{(2)}$. Skew-symmetry implies $a_{0} b=b_{0} a$ so that we may define

$$
a \bullet b=b \bullet a=a_{0} b,
$$

to form a commutative non-associative algebra on $V^{(2)}$ known as a Griess algebra. The Li-Z metric $\langle a, b\rangle$ on $V^{(2)}$ is an invariant bilinear form with

$$
\langle a \bullet b, c\rangle=\langle b, a \bullet c\rangle,
$$

for all $a, b, c \in V^{(2)}$.

\subsection{The automorphism group of a VOA}

The automorphism group Aut $(V)$ of a VOA $V$ consists of all elements $g \in G L(V)$ for which

$$
g Y(a, z) g^{-1}=Y(g a, z),
$$

with $g \omega=\omega$, the conformal vector. Thus the $L_{0}$ grading is preserved by $\operatorname{Aut}(V)$ and every Virasoro descendent of the vacuum is invariant under Aut $(V)$. Furthermore, the Li-Z metric is invariant with

$$
\langle g a, g b\rangle=\langle a, b\rangle,
$$

for all $a, b \in V$ and $g \in \operatorname{Aut}(V)$.

For VOAs with $\operatorname{dim} V^{(1)}>0$, then $\operatorname{Aut}(V)$ contains continuous symmetries $g=\exp \left(a_{0}\right)$ generated by elements of the Lie algebra $a \in V^{(1)}$. VOAs with $\operatorname{dim} V^{(1)}=0$ for which $V^{(2)}$ defines a Griess algebra are particularly interesting. Examples include the Moonshine Module $V^{\natural}$ of central charge $C=24$ with Aut $(V)=\mathbb{M}$, the Monster group [5]. In this case, $V^{\natural(2)}$ is the original Griess algebra of dimension $196884=1+196883$ where $V^{\natural(2)}$ decomposes into the $\mathbb{M}$ invariant Virasoro vector $\omega$ and an irreducible $\mathbb{M}$ representation of dimension $196883=47 \cdot 59 \cdot 71$. Other examples include a VOA with $C=23 \frac{1}{2}$ and $\operatorname{Aut}(V)=\mathbb{B}$, the Baby Monster where $\operatorname{dim} V^{(2)}=1+96255$ with an irreducible $\mathbb{B}$ representation of dimension $96255=3^{3} \cdot 5 \cdot 23 \cdot 31[7]$ and a VOA with $C=8$ and $\operatorname{Aut}(V)=O_{10}^{+}(2) \cdot 2$ and $\operatorname{dim} V^{(2)}=1+155$ with an irreducible $O_{10}^{+}(2)$ representation of dimension $155=5 \cdot 31$ [6]. In each case, $C$ corresponds to the values shown in Tables 2,3 and 4 .

\footnotetext{
${ }^{2}$ Non-degeneracy of the metric is not necessary here.

${ }^{3}$ The non-standard minus sign on the RHS of (4.13) is due to the normalization $\langle\mathbf{1}, \mathbf{1}\rangle=1$.
} 


\section{$5 \quad$ Lie algebras and Virasoro descendents}

\subsection{Quadratic Casimirs}

We now discuss the relationship between the structure of the Lie algebra $V^{(1)}$ and the Virasoro algebra described earlier. We initially follow a technique due to Matsuo [11, 12] for constructing a Casimir vector $\lambda^{(n)} \in V^{(n)}$ which is Aut $(V)$ invariant. The relationship with the Virasoro algebra follow provided $\lambda^{(n)} \in V^{(n)}(C, 0)$, the vacuum Virasoro descendents of level $n$. This occurs, for example, if $V^{(n)}$ contains no Aut $(V)$ invariants apart from the elements of $V^{(n)}(C, 0)$. Here we describe a new general method based on weaker assumptions for obtaining various constraints on $V^{(1)}$ by considering the expansion in various domains of a particular correlation function [15].

Consider a VOA with Li-Z metric with

$$
d=\operatorname{dim} V^{(1)}>0
$$

the dimension of the Lie algebra $V^{(1)}$. Let $V^{(1)}$ have basis $\left\{u_{\alpha} \mid \alpha=1, \ldots, d\right\}$ and dual basis $\left\{u^{\beta} \mid \beta=1, \ldots, d\right\}$ i.e. $\left\langle u^{\alpha}, u_{\beta}\right\rangle=\delta_{\beta}^{\alpha}$. For $n \geq 0$ define the quadratic Casimir vector [11]

$$
\lambda^{(n)}=u_{1-n}^{\alpha} u_{\alpha} \in V^{(n)},
$$

where $\alpha$ is summed. $\lambda^{(n)}$ is invariant under Aut $(V)$ following (4.14). We find from (4.10) and (4.11) that

$$
\lambda^{(0)}=-d \mathbf{1}, \quad \lambda^{(1)}=0 .
$$

Furthermore, using (4.8) it follows that for all $m>0$

$$
L_{m} \lambda^{(n)}=(n-1) \lambda^{(n-m)} .
$$

Suppose that $\lambda^{(n)} \in V^{(n)}(C, 0)$ i.e. $\lambda^{(n)}$ is a Virasoro descendent of the vacuum of level $n$. Then $\lambda^{(n)}$ can be determined exactly via (5.1) [11]. For example, if $\lambda^{(2)} \in V^{(2)}(C, 0)$ then $\lambda^{(2)}=k \omega$ for some $k$. Hence

$$
\left\langle\omega, \lambda^{(2)}\right\rangle=k\langle\omega, \omega\rangle=k \frac{C}{2} .
$$

But from (4.10) and (5.1) we find $\left\langle\omega, \lambda^{(2)}\right\rangle \mathbf{1}=L_{2} \lambda^{(2)}=\lambda^{(0)}=-d \mathbf{1}$. Thus, for $C \neq 0$

$$
\lambda^{(2)}=-\frac{2 d}{C} \omega
$$

$\lambda^{(2)}$ is necessarily singular at $C=0$ where $\operatorname{det} M^{(2)}=0$. Notice that (5.2) is just the familiar Sugawara construction of $\omega$ in the case that $V^{(1)}$ is a simple Lie algebra.

Assuming $\lambda^{(4)} \in V^{(4)}(C, 0)$ we similarly find

$$
\lambda^{(4)}=\frac{3 d}{C(5 C+22)}\left[4 L_{-2} L_{-2} \mathbf{1}+(2+C) L_{-4} \mathbf{1}\right],
$$

which is singular at $C=0,-22 / 5$ where $\operatorname{det} M^{(4)}=0$. 


\subsection{Rational correlation functions and the $V^{(1)}$ Killing form}

Let $a, b \in V^{(1)}$ and consider the following correlation function

$$
F(a, b ; x, y)=\left\langle a, Y\left(u^{\alpha}, x\right) Y\left(u_{\alpha}, y\right) b\right\rangle .
$$

Then we have

Proposition 1. $F(a, b ; x, y)$ is a rational function

$$
F(a, b ; x, y)=\frac{G(a, b ; x, y)}{x^{2} y^{2}(x-y)^{2}}
$$

where $G(a, b ; x, y)$ is bilinear in $a, b$ and is a homogeneous, symmetric polynomial in $x, y$ of degree 4.

Proof. The bilinearity of $G$ in $a, b$ is obvious. Locality (4.2) implies that $F(a, b ; x, y)$ is of the form $(5.4)[4,13]$ where $G(a, b ; x, y)$ is clearly symmetric. The degree and homogeneity follow from (4.1) and (4.7).

It is convenient to parameterize $G(a, b ; x, y)$ in terms of 3 independent coefficients $P(a, b)$, $Q(a, b), R(a, b)$ as follows

$$
G(a, b ; x, y)=P(a, b) x^{2} y^{2}+Q(a, b) x y(x-y)^{2}+R(a, b)(x-y)^{4} .
$$

Expanding the rational function $F(a, b ; x, y))$ in $\frac{x-y}{y}$ we find

$$
F(a, b ; x, y)=(x-y)^{-2}\left(P(a, b)+Q(a, b)\left(\frac{x-y}{y}\right)^{2}+\cdots\right),
$$

whereas expanding in $\frac{-y}{x-y}$ we find

$$
F(a, b ; x, y)=y^{-2}\left(R(a, b)+(2 R(a, b)-Q(a, b))\left(\frac{-y}{x-y}\right)+\cdots\right) .
$$

From the corresponding VOA expansions, $P, Q, R$ may be computed in terms of the Li-Z metric $\langle a, b\rangle$ and the Lie algebra Killing form

$$
K(a, b)=\operatorname{Tr}_{V^{(1)}}(\operatorname{ad}(a) \operatorname{ad}(b))=\operatorname{Tr}_{V^{(1)}}\left(a_{0} b_{0}\right),
$$

as follows:

Proposition 2. $P(a, b), Q(a, b), R(a, b)$ are given by

$$
\begin{aligned}
& P(a, b)=-d\langle a, b\rangle, \\
& Q(a, b)=K(a, b)-2\langle a, b\rangle, \\
& R(a, b)=-\langle a, b\rangle .
\end{aligned}
$$

Proof. Associativity (4.5) implies we may expand in $(x-y) / y$ to obtain

$$
\begin{aligned}
F(a, b ; x, y) & =\left\langle a, Y\left(Y\left(u^{\alpha}, x-y\right) u_{\alpha}, y\right) b\right\rangle=\sum_{n \geq 0}\left\langle a, Y\left(\lambda^{(n)}, y\right) b\right\rangle(x-y)^{n-2} \\
& =(x-y)^{-2} \sum_{n \geq 0}\left\langle a, \lambda_{0}^{(n)} b\right\rangle\left(\frac{x-y}{y}\right)^{n} .
\end{aligned}
$$


The leading term of (5.7) is determined by $\lambda_{0}^{(0)}=-d$ so that comparing with (5.5) we find $P(a, b)=-d\langle a, b\rangle$. Note that the next to leading term automatically vanishes since $\lambda_{0}^{(1)}=0$.

We may alternatively expand $F(a, b ; x, y)$ in $-y /(x-y)$ to find

$$
\begin{aligned}
F(a, b ; x, y) & =\left\langle a, Y\left(u^{\alpha}, x\right) e^{y L_{-1}} Y(b,-y) u_{\alpha}\right\rangle \\
& =\left\langle a, e^{y L_{-1}} Y\left(u^{\alpha}, x-y\right) Y(b,-y) u_{\alpha}\right\rangle \\
& =\left\langle e^{y L_{1}} a, Y\left(u^{\alpha}, x-y\right) Y(b,-y) u_{\alpha}\right\rangle \\
& =\left\langle a, Y\left(u^{\alpha}, x-y\right) Y(b,-y) u_{\alpha}\right\rangle \\
& =y^{-2} \sum_{m \geq 0}\left\langle a, u_{m-1}^{\alpha} b_{1-m} u_{\alpha}\right\rangle\left(\frac{-y}{x-y}\right)^{m},
\end{aligned}
$$

(respectively using skew-symmetry (4.4), translation (4.3), invariance of the Li-Z metric (4.9) and that $a$ is primary (2.2)). Comparing to the leading term of (5.6) we find using (4.10) that

$$
R(a, b)=\left\langle a, u_{-1}^{\alpha} b_{1} u_{\alpha}\right\rangle=-\left\langle a, u^{\alpha}\right\rangle\left\langle b, u_{\alpha}\right\rangle=-\langle a, b\rangle .
$$

Comparing the next to leading terms of (5.6) and (5.8) and using (4.11) and (4.12) we find

$$
2 R(a, b)-Q(a, b)=\left\langle a, u_{0}^{\alpha} b_{0} u_{\alpha}\right\rangle=-\left\langle u^{\alpha}, a_{0} b_{0} u_{\alpha}\right\rangle=-K(a, b) .
$$

We next show that if $\lambda^{(2)}$ is a vacuum Virasoro descendent then the Killing form is proportional to the Li-Z metric:

Proposition 3. Suppose that $\lambda^{(2)} \in V^{(2)}(C, 0)$. Then

$$
K(a, b)=-2\langle a, b\rangle\left(\frac{d}{C}-1\right) .
$$

Proof. If $\lambda^{(2)} \in V^{(2)}(C, 0)$ then (5.2) implies $\lambda_{0}^{(2)}=-\frac{2 d}{C} L_{0}$. The $n=2$ term in (5.7) is thus $-\frac{2 d}{C}\langle a, b\rangle$. Comparing to (5.5) we find

$$
Q(a, b)=-\frac{2 d}{C}\langle a, b\rangle
$$

and hence the result follows from Proposition 2.

Since the Li-Z metric is non-degenerate, it immediately follows from Cartan's condition that Corollary 1. The Lie algebra $V^{(1)}$ is semi-simple for $d \neq C$ and is solvable for $d=C$.

The Killing form (5.9) has previously arisen in the literature from considerations of modular invariance in the classification of $V^{(1)}$ for holomorphic self-dual VOAs in the work of Schellekens [14] for central charge $C=24$ and Dong and Mason [3] for $C=8,16,24$. A similar result also appears in [12] also based on a Casimir invariant approach.

We can repeat results of [14, 3] concerning the decomposition of $V^{(1)}$ into simple components for $d \neq C$. Corollary 1 implies

$$
V^{(1)}=\mathfrak{g}_{1, k_{1}} \oplus \mathfrak{g}_{2, k_{2}} \oplus \cdots \oplus \mathfrak{g}_{r, k_{r}},
$$

where $\mathfrak{g}_{i, k_{i}}$ is a simple Kac-Moody algebra of dimension $d_{i}$ (where $d=\sum_{i=1, \ldots, r} d_{i}$ ) and level

$$
k_{i}=-\frac{1}{2}\left\langle\alpha_{i}, \alpha_{i}\right\rangle
$$


where $\alpha_{i}$ is a long root of $\mathfrak{g}_{i, k_{i}}$ (so that $(a, b)_{i}=-\langle a, b\rangle / k_{i}$ defines a non-degenerate form on $\mathfrak{g}_{i, k_{i}}$ with normalization $\left.\left(\alpha_{i}, \alpha_{i}\right)_{i}=2\right)$. The dual Coxeter number $h_{i}^{\vee}$ for $\mathfrak{g}_{i, k_{i}}$ is then found from the Killing form $K\left(h_{\alpha_{i}}, h_{\alpha_{i}}\right)=4 h_{i}^{\vee}$. Hence (5.9) implies that for each simple component

$$
\frac{h_{i}^{\vee}}{k_{i}}=\frac{d}{C}-1
$$

This implies that a finite number of solutions for (5.10) exist for any pair $(C, d)$.

\subsection{Deligne's exceptional Lie algebras}

We next consider the expansion $(5.8)$ of $F(a, b ; x, y)$ to the next leading term assuming that $\lambda^{(4)}$ is also a vacuum Virasoro descendent so that (5.3) holds. This results in one further constraint on $P, Q, R$ leading to [15]

Proposition 4. Suppose that $\lambda^{(n)} \in V^{(n)}(C, 0)$ for $n \leq 4$. Then

$$
d(C)=\frac{C(5 C+22)}{10-C} .
$$

Note that $d(C)$ necessarily contains the independent factors of the level 4 Kac determinant $\operatorname{det} M^{(4)}$ since it must vanish for $C=0,-22 / 5$ for which the construction of $\lambda^{(4)}$ is impossible.

For positive rational central charge $C$ then $d(C)$ is a positive integer for only 21 different values of $C$. The remarks subsequent to Corollary 1 imply that the possible values are further restricted and that $V^{(1)}$ must be one of the Deligne exceptional simple Lie algebras [15]:

Proposition 5. Suppose that $\lambda^{(n)} \in V^{(n)}(C, 0)$ for $n \leq 4$ and that $C \in \mathbb{Q}_{+}$. Then $V^{(1)}$ is one of the simple Lie algebras $A_{1}, A_{2}, G_{2}, D_{4}, F_{4}, E_{6}, E_{7}, E_{8}$ for $C=1,2, \frac{14}{5}, 4, \frac{26}{5}, 6,7,8$ respectively, with Kac-Moody level $k=1$ and dual Coxeter number

$$
h^{\vee}(C)=6 \frac{2+C}{10-C}=\frac{d(C)}{C}-1 .
$$

$d(C)$ is precisely the original Vogel formula (3.1) with dual Coxeter number given by (5.11). The properties of Table 1 are now obvious since if a prime $p$ divides $d(C)$ then $p$ must divide one of the factors $C$ or $22+5 C$ of $\operatorname{det} M^{(4)}$. We note that results similar to Propositions 4 and 5 also appear in [12] but are based on a number of further technical assumptions.

We conclude this section with a result concerning the constraints arising from higher level Casimirs being vacuum descendents [15]:

Proposition 6. If $\lambda^{(n)} \in V^{(n)}(C, 0)$ for $n \leq 6$ then $V^{(1)}$ must be either $A_{1}$ or $E_{8}$ with level 1 . If $\lambda^{(n)} \in V^{(n)}(C, 0)$ for all $n$ then $V^{(1)}$ is $A_{1}$ level 1 .

\section{Griess algebras and Virasoro descendents}

We can now repeat the approach taken in the last section for a VOA with a Li-Z metric with $\operatorname{dim} V^{(1)}=0$ so that $V^{(2)}$ defines a Griess algebra. This was the original case considered by Matsuo [11] but was based on stronger assumptions (e.g. assuming the existence of a proper idempotent or that $\operatorname{Aut}(V)$ is finite). We give a brief description of the constraints arising from quadratic Casimirs being Virasoro descendents using a similar approach to the last section. Detailed proofs will appear elsewhere [15].

Let $\hat{V}^{(2)}=V^{(2)}-\mathbb{C} \omega$ denote the level 2 primary vectors of dimension

$$
d_{2}=\operatorname{dim} \hat{V}^{(2)}>0
$$


with basis $\left\{u_{\alpha}\right\}$ and dual basis $\left\{u^{\alpha}\right\}$. We define $\operatorname{Aut}(V)$ invariant quadratic Casimir vectors

$$
\mu^{(n)}=u_{2-n}^{\alpha} u_{\alpha} \in V^{(n)} .
$$

Then

$$
\mu^{(0)}=d_{2} \mathbf{1}, \quad \mu^{(1)}=0,
$$

and for $m>0$

$$
L_{m} \mu^{(n)}=(m+n-2) \mu^{(n-m)} .
$$

Consider the correlation function for $a, b \in \hat{V}^{(2)}$

$$
F(a, b ; x, y)=\left\langle a, Y\left(u^{\alpha}, x\right) Y\left(u_{\alpha}, y\right) b\right\rangle .
$$

Then we have

Proposition 7. $F(a, b ; x, y)$ is a rational function

$$
F(a, b ; x, y)=\frac{G(a, b ; x, y)}{x^{4} y^{4}(x-y)^{4}},
$$

where $G(a, b ; x, y)$ is bilinear in $a, b$ and is a homogeneous, symmetric polynomial in $x, y$ of degree 8.

In this case $G(a, b ; x, y)$ is determined by 5 independent coefficients. We use associativity (4.5) as in (5.7) to expand in $(x-y) / y$ so that

$$
F(a, b ; x, y)=(x-y)^{-4} \sum_{n \geq 0}\left\langle a, \mu_{0}^{(n)} b\right\rangle\left(\frac{x-y}{y}\right)^{n} .
$$

Similarly to (5.8), we expand in $-y /(x-y)$ to obtain

$$
F(a, b ; x, y)=y^{-4}\left(\langle a, b\rangle+0+\operatorname{Tr}_{\hat{V}^{(2)}}\left(a_{0} b_{0}\right)\left(\frac{-y}{x-y}\right)^{2}+\cdots\right) .
$$

Analogously to Propositions 2 and 3 and employing methods of [3] we find

Proposition 8. Suppose that $\mu^{(n)} \in V^{(n)}(C, 0)$ for $n \leq 4$. Then

1. $G(a, b ; x, y)$ is given by

$$
\begin{aligned}
\langle a, b\rangle & {\left[d_{2} x^{4} y^{4}+\frac{8 d_{2}}{C} x^{3} y^{3}(x-y)^{2}+\frac{4 d_{2}(44-C)}{C(5 C+22)} x^{2} y^{2}(x-y)^{4}\right.} \\
& \left.+2\left(x^{2}+y^{2}\right)(x-y)^{6}-(x-y)^{8}\right]
\end{aligned}
$$

2. $V^{(2)}$ has a non-degenerate trace form

$$
\operatorname{Tr}_{V^{(2)}}\left((a \bullet b)_{0}\right)=\frac{8\left(d_{2}+1\right)}{C}\langle a, b\rangle
$$

3. The Griess algebra $V^{(2)}$ is simple. 
$d_{2}$ and $C$ are related by the next Virasoro constraint in analogy to Proposition 4:

Proposition 9. Suppose that $\mu^{(n)} \in V^{(n)}(C, 0)$ for $n \leq 6$. Then

$$
d_{2}(C)=\frac{1}{2} \frac{(5 C+22)(2 C-1)(7 C+68)}{748-55 C+C^{2}} .
$$

The formula for $d_{2}(C)+1$ previously appeared in [11] subject to further assumptions. The appearance of the independent factors of $\operatorname{det} M^{(6)}$ in $d_{2}(C)$ follows as before. (The absence of a $C$ factor follows from the assumed decomposition $\left.V^{(2)}=\mathbb{C} \omega \oplus \hat{V}^{(2)}\right)$. If a prime $p$ divides $d_{2}(C)$ then $p$ must divide (at least) one of the independent factors $5 C+22,2 C-1$ or $7 C+68$ of $\operatorname{det} M^{(6)}$. This explains many of the prime divisor properties (but not all) for Tables 2,3 and 4 .

There are exactly 36 positive rational values for $C$ for which $d_{2}(C)$ is an integer. The simplicity of the Griess algebra can be expected to further restrict these possibilities. A full description of these "exceptional Griess algebras" and their possible realization in terms of VOAs would be of obvious interest [15].

Even stronger constraints on $\hat{V}^{(2)}$ follow if we assume that all $\operatorname{Aut}(V)$ invariants of $V^{(n)}$, $V^{(n)} / \operatorname{Aut}(V)$, are Virasoro descendents of the vacuum for $n \leq 6$ :

Proposition 10. Suppose that $V^{(n)} / \operatorname{Aut}(V) \subset V^{(n)}(C, 0)$ for all $n \leq 6$. Then, $\hat{V}^{(2)}$ is an irreducible representation of Aut $(V)$.

This concurs with the irreducible representation structure discussed in Section 4.4 with $d_{2}(C=24)=196883$ for the Monster, $d_{2}\left(C=23 \frac{1}{2}\right)=96255$ for the Baby Monster and $d_{2}(C=8)=155$ for $O_{10}^{+}(2)$.

The higher Casimirs lead to further constraints: if $\mu^{(n)} \in V^{(n)}(C, 0)$ for $n \leq 8$ or $n \leq 10$ then $C=24$ with $d_{2}=196883$. However, $\mu^{(n)} \in V^{(n)}(C, 0)$ for $n \leq 12$ is impossible. This concurs with the fact that the Moonshine module contains a Monster invariant primary vector of level 12 .

We can also consider the Casimirs $\nu^{(n)}=u_{3-n}^{\alpha} u_{\alpha} \in V^{(n)}$ constructed from a basis $\left\{u_{\alpha}\right\}$ of primary vectors of level 3

$$
\hat{V}^{(3)}=V^{(3)}-L_{-1} V^{(2)},
$$

of dimension $d_{3}=\operatorname{dim} \hat{V}^{(3)}>0$. We find

Proposition 11. Suppose that $\nu^{(n)} \in V^{(n)}(C, 0)$ for $n \leq 10$. Then $d_{3}(C)=p(C) / q(C)$ with

$$
\begin{aligned}
p(C)= & 5 C(5 C+22)(2 C-1)(7 C+68)(3 C+46)(5 C+3)(11 C+232), \\
q(C)= & 75 C^{6}-9945 C^{5}+472404 C^{4}-9055068 C^{3} \\
& +39649632 C^{2}+438468672 C+2976768 .
\end{aligned}
$$

All the factors of $\operatorname{det} M^{(10)}$ appear in $p(C)$ explaining the remarks concerning prime divisors in Table 2 where $d_{3}(24)=21296876=2^{2} \cdot 31 \cdot 41 \cdot 59 \cdot 71$ as obtains for the Moonshine Module. Finally, we also find

Proposition 12. Suppose that $V^{(n)} / \operatorname{Aut}(V) \subset V^{(n)}(C, 0)$ for all $n \leq 10$. Then $\hat{V}^{(3)}$ is an irreducible representation of $\operatorname{Aut}(V)$.

This agrees with the Monster irreducible decomposition of $V^{(3)}$ for the Moonshine Module where $\operatorname{dim} V^{(3)}=21493760=1+\operatorname{dim} \hat{V}^{(2)}+\operatorname{dim} \hat{V}^{(3)}=1+196883+21296876$.

\section{Acknowledgments}

The author thanks A. Matsuo and G. Mason for very useful discussions and H. Maruoka, A. Matsuo and H. Shimakura for generously making Ref. [12] available. 


\section{References}

[1] Borcherds R., Vertex algebras, Kac-Moody algebras and the Monster, Proc. Natl. Acad. Sci. USA 83 (1986), 3068-3071.

[2] Deligne P., La série exceptionnelle de groupes de Lie (The exceptional series of Lie groups), C. R. Acad. Sci. Paris Sér. I Math. 322 (1996), 321-326.

[3] Dong C., Mason G., Holomorphic vertex operator algebras of small central charge, Pacific J. Math. 213 (2004), 253-266, math.QA/0203005.

[4] Frenkel I., Huang Y-Z., Lepowsky J., On axiomatic approaches to vertex operator algebras and modules, Mem. Amer. Math. Soc. 104 (1993), no. 494.

[5] Frenkel I., Lepowsky J., Meurman A., Vertex operator algebras and the Monster, Academic Press, New York, 1988.

[6] Griess R.L., The vertex operator algebra related to $E_{8}$ with automorphism group $O^{+}(10,2)$, in The Monster and Lie algebras (1996, Columbus, Ohio State University), Math. Res. Inst. Public., Vol. 7, de Gruyter, Berlin, 1998.

[7] Hoehn G., Selbstduale Vertexoperatorsuperalgebren und das Babymonster, PhD Thesis, Bonn. Math. Sch. 286 (1996), 1-85.

[8] Kac V., Vertex operator algebras for beginners, University Lecture Series, Vol. 10, AMS, Boston, 1998.

[9] Kac V., Raina A.K., Bombay lectures on highest weight representations of infinite dimensional Lie algebras, World Scientific, Singapore, 1987.

[10] Li H., Symmetric invariant bilinear forms on vertex operator algebras, J. Pure Appl. Algebra 96 (1994), 279-297.

[11] Matsuo A., Norton's trace formula for the Griess algebra of a vertex operator algebra with large symmetry, Comm. Math. Phys. 224 (2001), 565-591, math.QA/0007169.

[12] Maruoka H., Matsuo A., Shimakura H., Trace formulas for representations of simple Lie algebras via vertex operator algebras, 2005, unpublished preprint.

[13] Matsuo A., Nagatomo K., Axioms for a vertex algebra and the locality of quantum fields, MSJ Memoirs, Vol. 4, Tokyo, Mathematical Society of Japan, 1999, hep-th/9706118.

[14] Schellekens A.N., Meromorphic $C=24$ conformal field theories, Comm. Math. Phys. 153 (1993), 159-185, hep-th/9205072.

[15] Tuite M.P., to appear. 